\title{
Unified Network Model for Adsorption- Desorption in Systems with Hysteresis
}

\author{
Pavol Rajniak and Miroslav Soós \\ Dept. of Chemical and Biochemical Engineering, Slovak Technical U niversity, 81237 Bratislava, Slovakia
}

Ralph T. Yang

Dept. of Chemical Engineering, U niversity of M ichigan, A nn A rbor, MI 48109

\begin{abstract}
The problem of equilibrium and kinetics for adsorption-desorption of condensable $v$ apors in porous media is studied experimentally and theoretically. For adsorption, the network model for diffusion based on pore blocking theory with percolation (in the network) added by effective medium approximation is further improved. A new predictive model based on properties of the Bethe lattices is proposed to account for the existence of liquid-filled "blind" pores that result in a decrease in the total diffusion rate. For desorption, a new "shell and core" (or shrinking core) representation of the network model is proposed. Information from adsorption-desorption equilibria is needed to compute the thickness of the shell in which desorption/evaporation occurs for concentrations higher than the percolation threshold. These models form a unified equilibrium-kinetics theory for gas-porous solid systems that exhibit hysteresis. The models are applied to the systems silica gel-water vapor and Vycor glass-nitrogen. Concentration-dependent Fickian diffusivities for these systems have been measured for both adsorption and desorption branches. The adsorption model successfully predicts the experimental data with a maximum in diffusivity. The desorption model correctly predicts the concentration dependence of diffusivity with a steep minimum at the percolation threshold.
\end{abstract}

\section{Introduction}

The problem of predicting diffusion rates of adsorbable vapors in porous media in the range of pressure where capillary condensation occurs is a significant one for the design and operation of adsorbers, dryers, catalytic reactors, membrane separators, and so on. Although many aspects of the diffusion/transport process are understood in principle (Sahimi, 1995), the important role of capillary condensation and evaporation has been largely neglected due to difficulties in separating the contributions from different transport mechanisms.

For adsorption and desorption of condensable vapors, the vapor in the adsorbent exists as adsorbed molecules on the solid surface, condensate in fine pores and as vapor in the voids. D uring adsorption at low partial pressures, monolayer

Correspondence concerning this article should be addressed to R. T. Y ang adsorption plays the major role. At higher partial pressures, the role of multilayer adsorption becomes important and simultaneously capillary condensation in the finer pores begins. Capillary condensation increases with increasing relative pressure, and eventually the entire pore volume becomes filled by capillary condensate. D uring desorption, as the relative pressure is reduced, systems in which capillary condensation occurs generally show hysteresis, that is, in a particular pressure range more vapor remains adsorbed during desorption than was adsorbed during the initial adsorption process. Classical explanations of hysteresis based on single pores (such as Everett, 1967; G regg and Sing, 1982) cannot satisfactorily explain some important experimental observations, such as the higher-order adsorption-desorption scanning curves. $M$ odels that treat the pore system as an interconnected network have been developed more recently. These models attribute hysteresis to pore blocking where the emptying of a 
large pore filled with capillary liquid has to be preceded by the emptying of its smaller neighbors (M ason, 1988; Parlar and $Y$ ortsos, 1988; Seaton, 1991; Li et al., 1991; Lilly et al., 1993; R ajniak and $Y$ ang, 1993, 1994). Hence, the primary desorption is a connectivity-related phenomenon. Such phenomena can be described best by percolation theory (Broadbent and $\mathrm{H}$ ammersley, 1957).

The percolation model for primary desorption is not in complete agreement with the experimental data shown in Figure $1 a$, because the initial part of the real desorption isotherm is not horizontal as described by the theory and shown in Figure 3a. There are various explanations for this fact: decompression of the liquid phase (M ason, 1988) and nucleation effects (Parlar and Y ortsos, 1989). Liu et al. (1993) carried out extensive $M$ onte Carlo simulations of the primary desorption and have shown that the finite size of the microparticles is important and the surface clusters must be taken into account. The desorption process is then related to invasion percolation (Wilkinson and Willemsen, 1983), the main difference being that here the invading phase (vapor in this case) enters from the whole surface of the system, rather than from just one face (Guyer and M cCall, 1996; Page et al., 1993).

The equilibrium behavior of capillary condensate is relatively well understood, but its dynamic behavior has not been studied in the works cited above. V arious experimental methods used to determine the mass transport rates in the capillary condensation regime ( $\mathrm{R}$ him and $\mathrm{H}$ wang, 1975; Tamon et
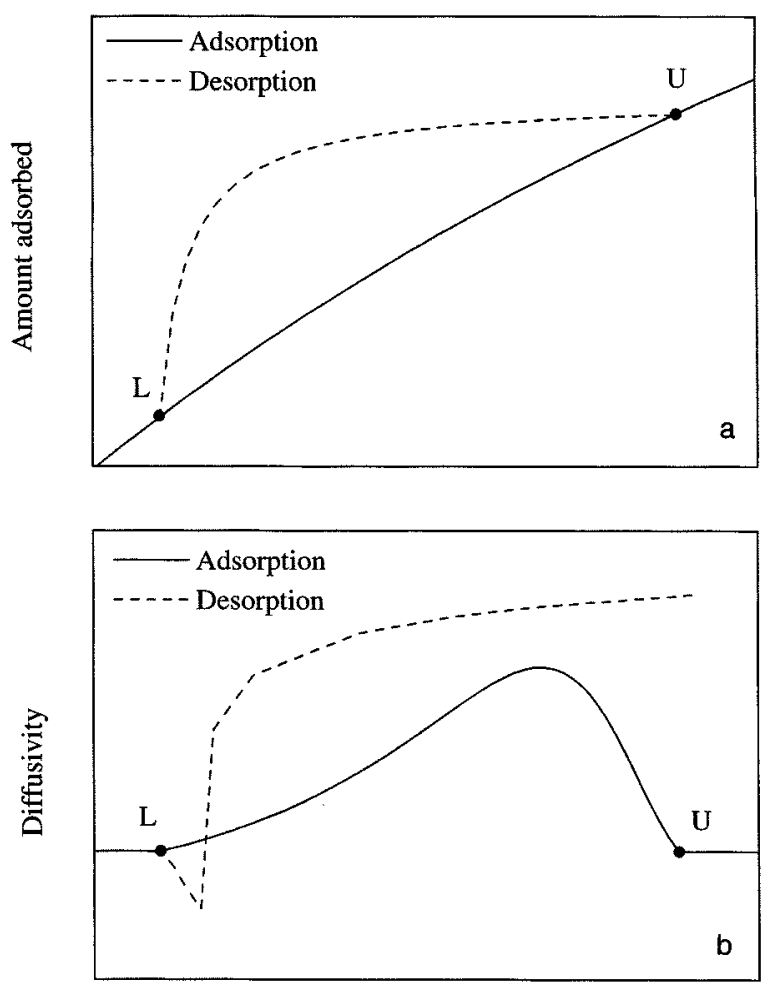

Relative Pressure

Figure 1. Experimental hysteresis dependent adsorption-desorption equilibria and kinetics.

(a) Equilibria; $L=$ lower limiting point, $U=$ upper limiting point of the hysteresis loop; (b) kinetics. al., 1981; Toei et al., 1983; E berly and Vosberg, 1965) and theoretical approaches explaining the experimental results (Carman, 1952; Flood et al., 1952; Gilliand et al., 1958; Kapoor et al., 1989) have appeared in the literature. Most of them are discussed in our previous work ( $R$ ajniak and $Y$ ang, 1996).

Like in adsorption-desorption equilibria, hysteresis has also been reported for the diffusion rates or kinetics in the capillary condensation region. Hysteresis in both equilibria and kinetics are illustrated, respectively, in Figures $1 \mathrm{a}$ and $1 \mathrm{~b}$. The concentration dependence of diffusivity exhibits a maximum during adsorption and a minimum during desorption. Interestingly, the maximum is located near the upper closure point (U) of the hysteresis loop (Chen and $Y$ ang, 1993), while the minimum appears near the lower closure point $(L)$. The adsorption and desorption branches coincide only in the region of surface diffusion, that is, at low partial pressures. Such concentration dependence is typically seen in gravimetric measurements for rates of adsorption and desorption when the results are fitted by the transient diffusion equation (Fick's second law) with a constant diffusivity (Kärger and R uthven, 1992; R ajniak and $Y$ ang, 1996). M ass transport in the porous material is a complex combination of gaseous diffusion, surface diffusion, capillary condensation/evaporation, and flow of liquid condensate. Evaluation of such a complicated process using diffusion equation with a constant diffusivity is only a mathematical simplification for the purpose of comparing rates at various concentrations during adsorption and desorption. Literature reports on hysteresis in the kinetics in adsorption-desorption include the study of capillary condensation flow of toluene in Vycor glass (A beles et al., 1991), gravimetric measurements of the kinetics of isothermal adsorption and desorption of isopropanol in $\mathrm{V}$ ycor glass ( $\mathrm{H}$ aynes and Miller, 1982), multilayer diffusion and capillary condensation of propylene in supported alumina films (U hlhorn et al., 1992), permeabilities in $\mathrm{V}$ ycor glass (Lee and $\mathrm{H}$ wang, 1986), and isothermal transport of liquids in partially saturated packed beds of glass spheres (Büssing et al., 1996).

Literature reports that are of particular interest to this work are the theory on preparation of supported catalysts ( $\mathrm{N}$ eimark et al., 1981), moisture transport in microporous substances (Radjy, 1974), study of drying by NMR imaging (Maneval et al., 1991), and determination of moisture diffusivity in porous media using concentration profiles (Pel et al., 1996). The models used in these studies represent the classic continuum approach (Sahimi et al., 1990). M ost of these works are restricted to the idealized case of a single pore size. The complexities caused by the pore-size distribution and by the network effects were not considered. In the continuum models, the porous medium is treated as a continuum within which the properties of the fluid and solid phases are defined as smooth functions of time and positions. Continuum models are often not adequate for systems consisting of phases that differ appreciably from one another in their effective properties (Sahimi et al., 1990).

There have been only a few studies on kinetics using network models where phase changes are included (D aian, 1992; Y ortsos et al., 1993; Prat, 1993, 1995; L aurindo and Prat, 1996; $\mathrm{Li}$ and $\mathrm{Y}$ ortsos, 1995; R ajniak and $\mathrm{Y}$ ang, 1996). D aian (1992) examined various methods for computing moisture diffusion rates. The interactions between vapor diffusion and liquid 
transport are discussed based on the network theory. Some experimental results seem to indicate that the actual role of vapor diffusion is strongly influenced by its interaction with the liquid phase through condensation and evaporation. Prat and coworkers studied evaporation in porous media (Prat, 1993, 1995; Laurindo and Prat, 1996). They conclude that evaporation belongs to the invasion percolation type. The main difference between evaporation and standard invasion percolation lies in the erosion of the disconnected liquid clusters that form as a result of the growth of the invading vapor phase. As phase change takes place at the boundary of these disconnected clusters, they can be invaded in evaporation while they are generally considered as trapped in standard invasion percolation. Experimental and simulated phase distributions (L aurindo and Prat, 1996) for the case with stabilizing gravity forces show a sharp boundary between the liquid and vapor phases.

In our recent work ( $R$ ajniak and $Y$ ang, 1996), a network model was formulated for predicting effective Fickian diffusivities of condensable vapors in porous media where capillary condensation and adsorption-desorption hysteresis occur. The model unifies the equilibrium theory based on the pore-blocking interpretation of hysteresis in the interconnected network of pores and the percolation model of mass transport in the network with randomly interspersed regions for capillary condensation and surface flow. In the present work, we briefly summarize the main concepts of the equilibrium theory for adsorption-desorption systems with hysteresis (M ason, 1988; Rajniak and Y ang, 1993, 1994) based on the pore-blocking theory and percolation theory in the Bethe tree network. Next, the network model for both adsorption and desorption kinetics is developed for the idealized case of the infinite media. Modifications of the theory necessary for real systems with finite dimensions of the network are then proposed. For adsorption, a modification is proposed for the computation of "blind" liquid-filled pores. The derivation is based again on the Bethe tree approximation of the pore network structure. The only parameter in the proposed model is connectivity, which can be obtained independently. The improved model predicts the concentration dependence of the effective diffusivity for systems with a maximum diffusivity. For desorption kinetics, a model is proposed for computing the descending branch of the concentration dependence of effective diffusivity for relative pressures higher than that corresponding to the percolation threshold. The proposed model is based on the "shrinking core" (Sahimi et al., 1990; Lee and Aris, 1985; Brunovská et al., 1985; Markos et al., 1987). In order to compare the predictions of the proposed models with experimental data, a complete set of concentration-dependent equilibrium-diffusion data is obtained for the system $\mathrm{V}$ ycor glass-nitrogen.

\section{Theoretical Considerations}

We consider the adsorption-desorption process of a condensable vapor in a porous adsorbent. In Figure la the adsorption-desorption equilibria for the process are shown. At relative pressures below point $L$, the lower closure point of the hysteresis loop, only surface adsorption occurs. The position of point $\mathrm{L}$ is characteristic of each sorbate-sorbent pair (such as, Naono and Hakuman, 1993; Burgess et al., 1989).
$M$ ass transport rates in this region are contributed by gaseous diffusion and surface diffusion. M ass transport rates in this region are the same for both adsorption and desorption, and the study of the kinetics in this region is out of scope of this work (Kärger and R uthven, 1992; Y ang, 1987).

A bove point $U$, the upper closure point of the hysteresis loop, all pores become filled with capillary condensate. The position of point $U$ depends on the pore-size distribution of the adsorbent. If point $U$ lies below the saturation pressure of the adsorptive, then it is presumed that the solid has no pores of radii greater than that corresponding to the closure point. Adsorption beyond this pressure is related to the change of curvature of menisci freely accessible to the vapor and the compression of liquid condensate in the pores ( $\mathrm{L}$ iu et al., 1993). On the other hand, when decreasing the pressure in the region above point $U$, a negative pressure is produced and this decompression reduces the density of liquid condensate (M ason, 1988). Transport of liquid condensate in the region above point $U$ can then occur only by hydraulic pressure, and, under usual conditions of adsorption experiments, this flow is much smaller than the capillary condensate flow. During adsorption at point $L$, the condensation pressure is reached in the finest pores and the pores are filled with a liquidlike phase. The extent of capillary condensation increases with increasing pressure, and, at point $U$, the entire pore volume is filled by capillary condensate.

In this study we are interested in the mass transport rate in the region of capillary condensation, that is, in the region between the limiting points of the main hysteresis loop $L$ and U.

\section{Adsorption-desorption equilibria for infinite systems}

The pore blocking theory ( $M$ ason, 1988; R ajniak and $Y$ ang, 1993, 1994) was developed directly for the capillary condensation domain, and it describes the adsorption-desorption equilibria for relative pressures $\mathrm{x}^{\mathrm{L}}<\mathrm{x}<\mathrm{x}^{\mathrm{U}}$. Following the pore blocking theory, a porous material consists of a number of pores connected together in a network in which we distinguish sites (pore bodies) from bonds (pore throats). The individual sites are connected via bonds. The average number of bonds to each site is defined as connectivity $C$. The characteristic dimension of the site or of the bond $r$ can be related to the macroscopic Kelvin equation for capillary condensation. Thus, at any value of relative pressure $x$, the process may be uniquely parametrized by the characteristic dimension $r$. A nother important function is $q$, the probability that the site is filled by capillary condensate at relative pressure $x$. Similarly $p$ is the probability that the bond is filled at the relative pressure $x$. U nder the assumptions that the capillary condensation phenomenon controls the equilibrium of the sorption process between points $L$ and $U$, and that the volume of capillary condensed liquid is primarily associated with the cavities, the adsorption process is represented by an increase of $q=0$ at $x=x^{L}$ to $q=1$ at $x=x^{U}$. On the other hand, the desorption process is represented by a decrease of $\mathrm{p}=1$ at $\mathrm{x}=\mathrm{x}^{\mathrm{U}}$ to $\mathrm{p}=0$ at $\mathrm{x}=\mathrm{x}^{\mathrm{L}}$.

The probabilities $p$ and $q$ are related through connectivity C by (M ason, 1988; R ajniak and $Y$ ang, 1993)

$$
q=p^{c}
$$


To relate $q$ and $p$ to $a$, the amount adsorbed on the sorption isotherms, the variable $\mathrm{S}$ must be introduced. $\mathrm{S}$, the fraction of pores filled, is related to the adsorbed amount by the expression

$$
S=\frac{a-a^{L}}{a^{U}-a^{L}}
$$

The amount adsorbed a during adsorption or desorption is a function of the relative pressure $\mathrm{x}$ via the adsorption or desorption isotherm, that is,

$$
\begin{aligned}
& a_{A}=f(x) \\
& a_{D}=f(x)
\end{aligned}
$$

For the primary adsorption process (the subscript $A$ is for primary adsorption and the subscript $D$ for primary desorption), the fraction of pores filled by capillary condensation is

$$
\mathrm{S}_{\mathrm{A}}=\mathrm{q}
$$

It is worth noting that $S_{A}$ is not dependent on connectivity $C$. Pore blocking does not play any role in adsorption equilibria. When a porous material is filled by adsorption, all of the pores are equally accessible. Even if the pore becomes isolated from the bulk vapor, it can still fill by condensing vapor from adjacent pores that can then refill from the bulk vapor.

For primary desorption, the hysteresis of capillary condensed vapor can be explained by pore-blocking effects, where a pore cannot empty until at least one of its neighbors has emptied. This effect depends in principle on the interconnections and the interconnectedness of the pore network. The model frequently used for the interconnectedness is the B ethe tree shown in Figure 2. The advantage of using the Bethe tree is that the description of its behavior can be carried out

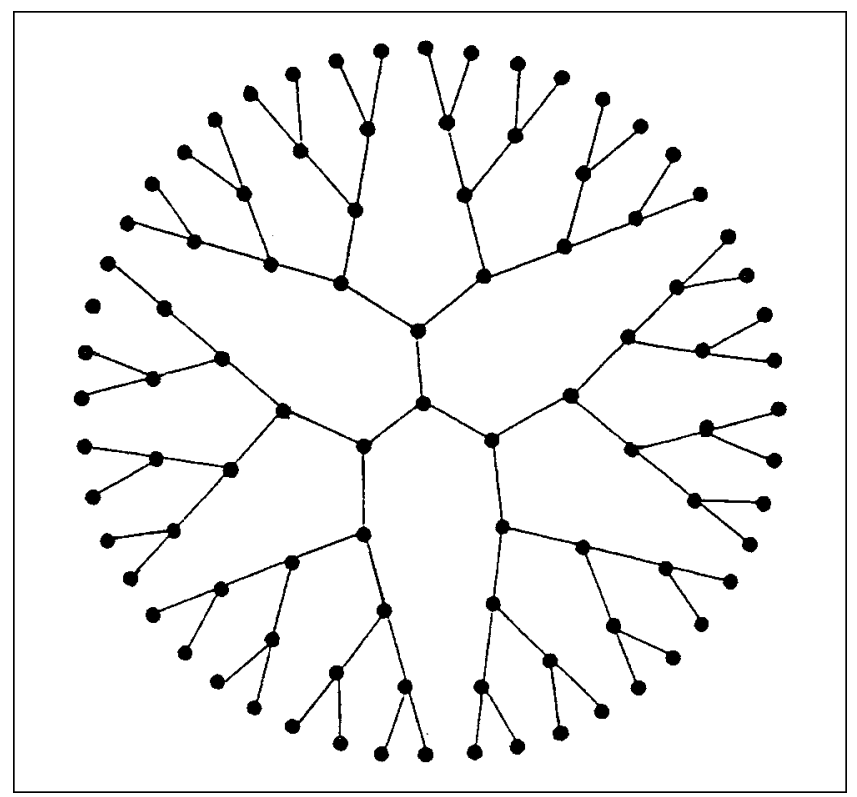

Figure 2. Bethe lattice with connectivity $C=3$. analytically. Bethe trees can give simplified expressions for several properties of the porous medium, while retaining most features of percolation theory (Sahimi, 1993; Kainourgiakis et al., 1998).

The analysis given here uses percolation theory (Stauffer and A harony, 1992; Sahimi, 1994, 1995) with the restriction to a Bethe tree network. During the primary desorption process, whether a site remains full or empty depends on whether one of its bonds is connected to the vapor and at the same time can also be emptied. At some stage during primary desorption, at some value of $p$, the probability that a bond into a site is connected to the vapor is $v$. The fraction of the pores filled during primary desorption $S_{D}$ at probabilities $\mathrm{p}$ and $v$ can be computed via Eqs. 5 and 6 derived by M ason (1988)

$$
\mathrm{S}_{\mathrm{D}}=(v \mathrm{p}+1-v)^{\mathrm{C}}
$$

where

$$
(v \mathrm{p}+1-v)^{(\mathrm{C}-1)}=1-v
$$

Combining Eqs. 1-6 and knowing connectivity C, we can compute for any relative pressure $\mathrm{x}$ the amount adsorbed during desorption $a_{A}$, or during desorption $a_{D}$, and the corresponding fractions of pores filled $S_{A}$ or $S_{D}$, and the probabilities $q$ and $p$.

Figure $3 a$ shows the theoretical primary desorption isotherm computed from $\mathrm{Eqs}$. $1-6$ assuming $\mathrm{C}=3$. The ini-
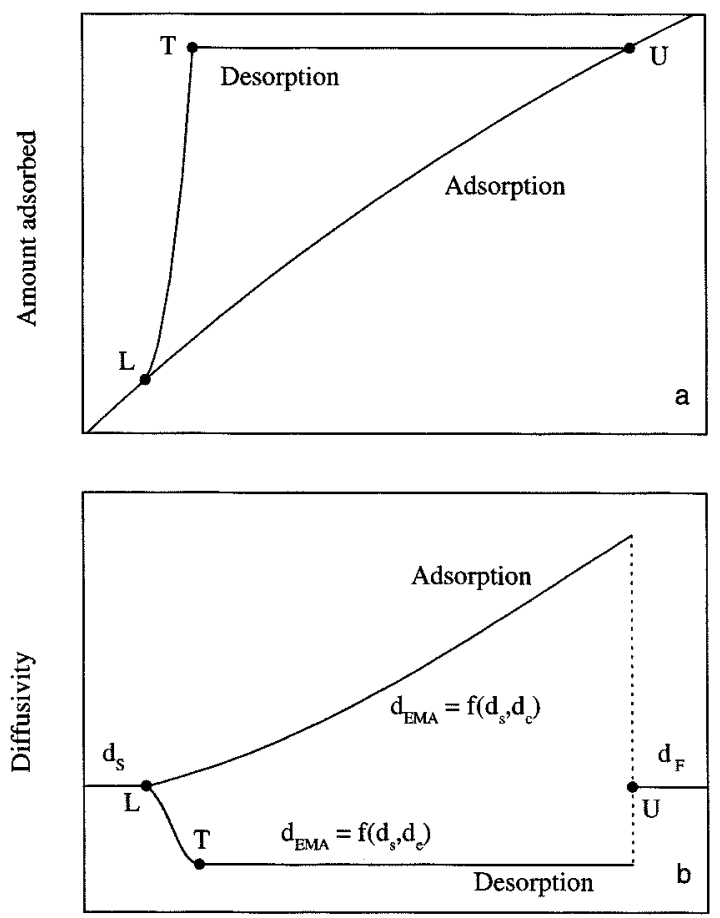

Relative Pressure

Figure 3. Theoretical hysteresis-dependent adsorptiondesorption equilibria and kinetics.

(a) Equilibria, $\mathrm{T}=$ percolation threshold; (b) kinetics 
tial part of the theoretical desorption isotherm (between points $U$ and $T$ ) is a horizontal line. In fact, the theoretical relations 5 and 6 give values $S_{D}=1$ for all values of $p$ greater than $\mathrm{p}^{\top}$, where

$$
p^{\top}=\frac{(C-2)}{(C-1)}
$$

is the value of probability $p$ at the percolation threshold $T$ of the desorption process.

Along this desorption branch, there is theoretically no desorption. Desorption should begin only at point $\mathrm{T}$, the percolation threshold for primary desorption, when there is a sufficient number of bonds in which the adsorbate is below its condensation pressure (and thus is present as either metastable liquid or vapor) and, consequently, a sufficient number of vapor connections in the porous network and desorption from the bulk of the adsorbent can start.

The primary adsorption process in the capillary condensation region can be treated as a classic site percolation problem. Pores with different dimensions are randomly distributed in the pore space. In this case pore sites of the network are filled by capillary condensate with probability $q$ and partially filled by surface adsorption with probability $1-$ q. However, percolation characteristics of the primary adsorption are not important for description of the equilibrium behavior of the process, because whether a particular site is filled by capillary condensation depends only on its characteristic dimension. The percolation properties of the primary adsorption are of particular interest for the kinetic behavior of the process.

The primary desorption equilibrium problem belongs to the group of bond percolation problems, because the emptying of the pore sites is determined by the bonds connections. Connectivity of the network plays a dominant role in the description of the process. The theoretical model using Bethe tree is developed for the infinite system and the clusters at the surface of the system cannot be taken into account. Between points $T$ and $U$ starting with a filled system, $p$ is progressively reduced until at the critical value $p^{\top}$, the system starts to empty. This occurs at the critical percolation probability $p^{\top}$. For an infinite system, there is no desorption until the percolation threshold is reached and the desorption isotherm has a discontinuity in slope at the threshold.

\section{Adsorption-desorption kinetics for infinite systems}

$M$ ass transport in the capillary condensation region is a complex phenomenon. The conditions under which capillary condensation occurs are also those under which significant surface diffusion is expected. Study of this phenomenon is therefore complicated by the difficulty of separating the contributions from the various transport mechanisms. Fortunately, vapor-flow contribution is found to be negligible under these conditions. Vapor-phase transport is in this case usually orders of magnitude smaller than the surface flow because of the relatively small amount of molecules in the vapor phase compared to that in the adsorbed phase ( $\mathrm{H}$ aynes and Miller, 1982; A beles et al., 1991). As soon as a pore is filled with condensate during adsorption, the vapor flux through that pore is cut off and transport then depends on surface diffusion together with any bulk flow induced by the capillary forces. It is not generally recognized that the capillary suction is a contributor leading to the mass-transfer amplification (Weisz, 1975; L ee and H wang, 1986; A beles et al., 1991; Kärger and Ruthven, 1992; Radjy, 1974; R ajniak and Y ang, 1996). A ny capillary condensate volume elements will create a short-circuit effect leading to a reduction in the length of the diffusion path and a corresponding increase in the effective diffusivity. As a consequence, capillary condensation generally increases the total mass transport rate. As the relative pressure is increased, the number of pores that are still not filled with condensate decreases rapidly, and at point $U$, all pores are filled with capillary condensate. So, the first effect of the capillary condensation is to increase the mass transport rate when some pores are filled with condensate. For theoretical (infinite) media, the total mass transport rate during adsorption will increase in the capillary condensation region with an increasing number of pores being filled by capillary condensation, as shown in Figure $3 b$.

If the upper closure point $U$ lies below $x=1$, we can expect a decrease of the total mass transport rate for relative pressures $\mathrm{x}^{\mathrm{U}}<\mathrm{x}<1$, because in this region all pores are filled with capillary condensate and the transport is controlled by the flow of liquid condensate. For $x>x^{U}$, there exists a discontinuity in the concentration dependence of diffusivity that drops abruptly to the value characteristic of the system when the whole sample is filled with condensate. In this region, the transport is controlled by the liquid flow of capillary condensate during the compression of the condensate in the sample and flattening of the menisci. Such discontinuity is an artifact of the model for the theoretical infinite system of connected pores; and it does not exist in real systems. If the upper closure point $U$ is at $x=1$, capillary condensation accelerates the mass transport in the whole concentration range above point $\mathrm{L}$. Theoretical prediction of diffusivity during adsorption is discussed in detail in our previous work ( $R$ ajniak and Y ang, 1996).

The physical situation is quite different for the case of desorption or evaporation of the condensed adsorbate. While the condensation generally increases the mass transport rate, evaporation is a very slow process and one can expect a decrease in the total mass transport rate with an increasing role by evaporation from pores. For an infinite medium, there is no desorption between points $U$ and $T$ and diffusivity is zero in this region, as shown in Figure $3 b$. At the percolation threshold $\mathrm{T}$, desorption starts and the diffusivity increases from zero. At point $L$, the two branches of concentrationdependent diffusivities coincide.

Theoretical models for infinite media successfully predict some qualitative features of the concentration dependence of diffusivity, that is, a rise in diffusivity during adsorption between points $L$ and $U$ and a rise in diffusivity between points $T$ and $L$ during desorption. However, the models cannot predict extremes on both dependencies, that is, a maximum for adsorption and a minimum for desorption, which are typical for the real finite systems.

O ne of the simplest methods for estimating the effective transport properties of disordered media is the effective medium approximation (EMA), which is a phenomenological method by which a disordered medium is replaced with a hypothetical homogeneous one represented by unknown 
physical constants (K irkpatrick, 1971; Sahimi, 1993). EM A thus transforms a many-body system into a one-body problem by which effective transport coefficients can be obtained.

The problem of mass transport in the porous adsorbent with randomly distributed fractions of pores filled by capillary condensation and by surface adsorption is similar to the problem of diffusion in a bi-disperse medium (Benzoni and Chang, 1984; Burganos and Sotirchos, 1987). Generally, a discrete multimodal distribution of pore diffusivities has the form

$$
f(d)=\sum_{i=1}^{n} f_{i} \delta\left(d-d_{i}\right)
$$

where $\delta$ is the Delta function and $d_{i}$ is the diffusivity of the pore of the ith kind (such as, $d_{i}=d_{s}$ for the pore partially filled by surface adsorption and $d_{i}=d_{c}$ for the pore filled by capillary condensation) and $n$ is the number of different fractions of pores. The EMA equation for the Bethe lattice with connectivity C (Stinchcombe, 1974; Heinrichs and Kumar, 1975; Sahimi, 1993) reduces to the summation

$$
\sum_{i=1}^{n} f_{i} \frac{d_{i}-d_{E M A}}{d_{i}+(C-2) d_{E M A}}=0
$$

where $n$ is the number of different fractions of pores, $d_{E M A}$ is the effective medium diffusivity, and $d_{i}$ is the diffusivity of the pore of type $i$.

The value of $d_{E M A}$ computed from the effective medium equation ( $\mathrm{Eq} .9$ ) is related to the effective diffusivity of the whole network $D_{E M A}$ (Stinchcombe, 1974) by

$$
D_{E M A}=C \frac{(C-2)}{(C-1)} d_{E M A}
$$

The following assumptions are made for all ensuing models:

(1) For relative pressures $x<x^{L}$, all pores of the porous medium are partially filled by surface adsorption, while for $\mathrm{x}>\mathrm{x}^{\mathrm{U}}$ all pores are filled by capillary condensation. For $\mathrm{x}^{\mathrm{L}}$ $<\mathrm{x}<\mathrm{x}^{\mathrm{U}}$, that is, in the hysteresis domain, the pores are partially filled by surface adsorption as well as capillary condensation.

(2) The fraction of pores filled by capillary condensation is $\mathrm{S}$ and that by surface adsorption is $1-\mathrm{S}$. For primary adsorption $S_{A}$ is given by Eq. 4 and for primary desorption $S_{D}$ is given by $\mathrm{E}$ qs. 5 and 6 .

(3) The symbol $d_{s}$ was used for the diffusivity in the pore site that is partially filled by surface adsorption and $d_{c}$ for the diffusivity in the pore site that is filled by capillary condensation during adsorption in the hysteresis region. (M ass transport occurs in pores containing condensate by pressuredriven flow, rather than diffusion, and the use of $d_{C}$ is a formal simplification.) Similarly, we will use the symbol $d_{E}$ for the diffusivity in the pore site that is filled by capillary condensation during desorption in the hysteresis region. We will use the symbol $d_{F}$ for the diffusivity in the pore site that is filled by capillary condensation in the region of flow of capillary condensate, that is, for relative pressures $\mathrm{x}>\mathrm{x}^{\mathrm{U}}$.
(4) We will assume $d_{E}<d_{S}<d_{C}$ (that is, evaporation is slower than surface adsorption which is slower than capillary condensation), and also $d_{F}<d_{C}$ (that is, flow of capillary condensate is slower than diffusion of capillary condensate).

(5) The bonds (pore throats and windows) between the pore sites do not contribute significantly to the total volume and the volume of capillary condensed liquid is associated only with the pore sites.

Based on the foregoing discussion, the model for the adsorption kinetics in the infinite media is given by Model A 1 as follows:

\section{Model A1:}

For $\mathrm{x} \leq \mathrm{x}^{\mathrm{L}}$ :

$$
f_{1}=1 \quad f_{2}=0 \quad d_{E M A}=d_{S}
$$

For $\mathrm{x}^{\mathrm{L}}<\mathrm{x}<\mathrm{x}^{\mathrm{U}}$ :

$$
\begin{gathered}
f_{1}=1-S_{A} \quad f_{2}=S_{A} \quad d_{1}=d_{S} \quad d_{2}=d_{C} \\
d_{E M A} \text { given by solution of E } q .9 \text { for } n=2
\end{gathered}
$$

For $x \geq x^{U}$ :

$$
f_{1}=0 \quad f_{2}=1 \quad d_{E M A}=d_{F}
$$

The dependence of the effective diffusivity for Model A 1 is shown in Figure $3 b$. M odel $A 1$ is equivalent to $M$ odel 2 discussed in our previous article ( $R$ ajniak and $Y$ ang, 1996), where the theoretical dependencies for various values of connectivity are also shown. The dependence of diffusivity contains a maximum at $\mathrm{x}=\mathrm{x}^{\mathrm{U}}$. For $\mathrm{x}>\mathrm{x}^{\mathrm{U}}$, there exists a discontinuity in the concentration dependence of diffusivity that decreases abruptly to the value $d_{F}$.

The model for desorption kinetics in the infinite media that is discussed in the present analysis (Model D1) is summarized as follows:

\section{Model D 1:}

For $x \geq x^{U}$ :

$$
f_{1}=0 \quad f_{2}=1 \quad d_{E M A}=d_{F}
$$

For $\mathrm{x}^{\top}<\mathrm{x}<\mathrm{x}^{\mathrm{U}}$ :

$$
f_{1}=0 \quad f_{2}=S_{D}=1 \quad d_{E M A}=0
$$

For $\mathrm{x}^{\mathrm{L}}<\mathrm{x}<\mathrm{x}^{\top}$ :

$$
\begin{gathered}
f_{1}=1-S_{D} \quad f_{2}=S_{D} \quad d_{1}=d_{S} \quad d_{2}=d_{E} \\
d_{E M A} \text { given by solution of Eq. } 9 \text { for } n=2
\end{gathered}
$$

For $\mathrm{x} \leq \mathrm{x}^{\mathrm{L}}$ :

$$
f_{1}=1 \quad f_{2}=0 \quad d_{E M A}=d_{S}
$$



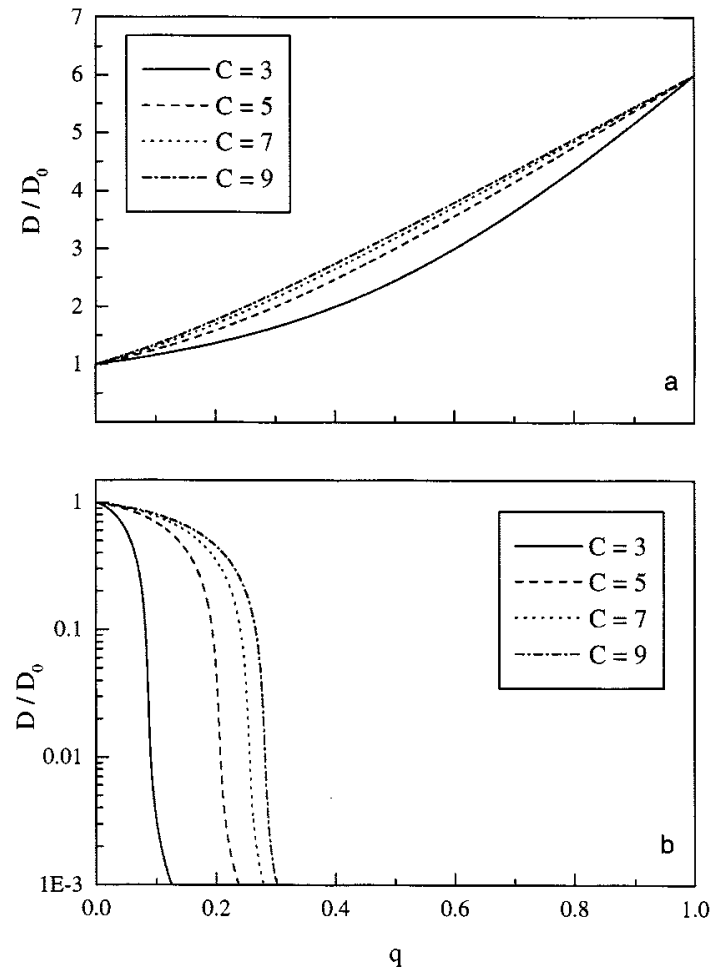

Figure 4. Theoretical dependencies of diffusivity on pore-filling ( $q$ ) for various connectivity (C).

For (a) adsorption (M odel A 1); (b) desorption (M odel D 1).

The theoretical dependence of the effective diffusivity based on Model D1 is shown in Figure $3 b$. The model predicts a decreasing diffusivity between points $L$ and $T$, which is observed also for real systems. Between points $T$ and $U$, the diffusivity is zero indicating no desorption or mass transfer in this region.

In Figure 4 the theoretical dependencies of effective diffusivity for various connectivities are shown for both adsorption and desorption. M odels $A 1$ and D 1 successfully predict the basic features of experimental results, that is, a maximum in diffusivity during adsorption and a minimum during desorption. H owever, there exist artifacts in the models which need further discussion, that is, discontinuity in the concentration dependence during adsorption and zero diffusivity in the concentration dependence during desorption.

\section{Adsorption-desorption equilibria for finite systems}

From the viewpoint of the equilibrium behavior, the primary adsorption process in the capillary condensation region can be treated as a classic site percolation problem. The pore sites of the network are filled by capillary condensate with probability $q$ and partially filled by surface adsorption with probability $1-q$. As discussed earlier, whether a particular site is filled by capillary condensation depends only on its characteristic dimension, while the connectivity of the pores is irrelevant, and the primary adsorption for the real systems, that is, porous media with finite dimensions, is the same as that for the infinite systems described by Eqs. 2, 3a, and 4.

Disagreement between theory and experiment exists for the case of real primary desorption, since the real desorption

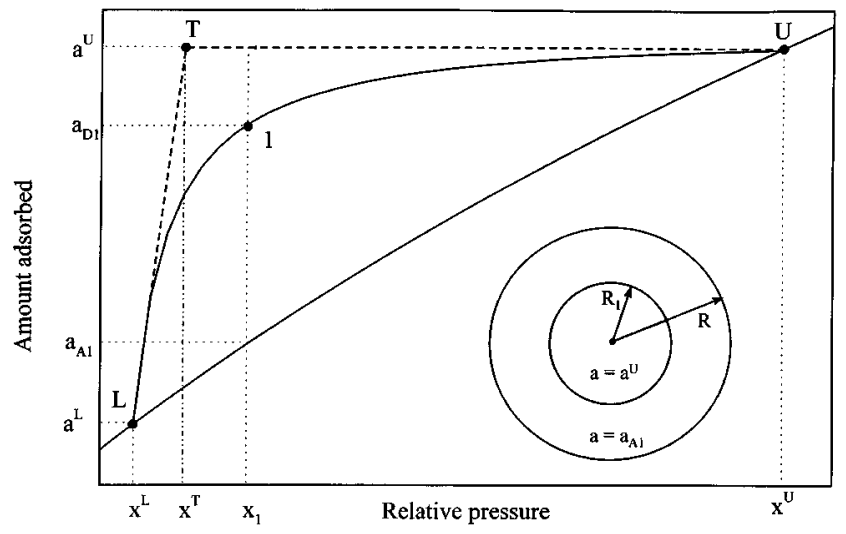

Figure 5. Desorption equilibria for finite systems.

A mount adsorbed in the shell $\mathrm{a}_{\mathrm{A} 1}$ and in the core $\mathrm{a}^{\mathrm{U}}$ at point 1 on the experimental primary desorption curve where the amount adsorbed is $a_{D 1}$ (inset) shell and core of the microparticle for amount adsorbed $\mathrm{a}_{\mathrm{D} 1}$ (see also Eq. 11).

isotherm is not horizontal and desorption does occur. Various explanations for this fact were discussed in our previous work (Rajniak and $Y$ ang, 1994) and empirical correction functions were proposed for computation of the real amount adsorbed during primary desorption, as well as during higher-order desorption processes. H owever, most of the theoretical and experimental studies indicate that the finite size of the microparticles of the porous material and corresponding desorption from the surface of the microparticles is the most important reason of the disagreement. The real primary desorption can be described by various relations ( $R$ ajniak and Y ang, 1993, 1994). H owever, use of either Langmuir or D ubinin or other kind of adsorption equilibria relationship for the hysteresis-dependent desorption is only a mathematical simplification.

Some properties of the Bethe trees make them unsuitable for evaluating surface effects of the real media. The fraction of the sites of the Bethe tree that are on its surface is given by $(C-2) /(C-1)$. This means that, in a Bethe lattice, most of the sites (or bonds) are on the surface, whereas, in reality, this fraction is small. So the Bethe tree model of the pore structure can be used only for the core of the microparticle and another approach has to be used for the surface.

In this work, a new approach based on mass balance of the adsorbed material in the particle will be used to evaluate the amount adsorbed during primary desorption. We assume that between points $T$ and $U$ in Figure 5 , as the pressure decreases, the condensed adsorbate is able to vaporize only from some pores near the outer surface of the microparticle, and then from some of the pores adjacent to those vapor filled pores, so that clusters of vapor filled pores (or surface clusters) grow on the outer surface of the microparticle. The vapor phase increasingly penetrates the surface layer of the microparticle. At the same time, the bonds in the bulk of the microparticle form clusters of bonds in which the adsorbate is below its condensation pressure but without access to the vapor phase. Only at the percolation threshold, the bonds in the bulk of the microparticle form a percolating cluster that has access to the outer surface, and desorption from the bulk of the microparticle can start. The decrease in the amount 
adsorbed between points $U$ and $T$ on the real primary desorption isotherm is a consequence of the desorption from the surface cluster of the microparticle of the porous material. Next we assume that there is a distinct boundary between the surface shell (that is partially desorbed) and the bulk core (that is completely filled) for the microparticle (see Figure 5 inset). A t relative pressure $\mathrm{x}\left(\mathrm{x}^{\top}<\mathrm{x}<\mathrm{x}^{\mathrm{U}}\right)$, the core is completely filled with liquid condensate, that is, the fraction of the pores filled in the core is $S=1$ and the amount adsorbed is $\mathrm{a}^{\mathrm{U}}$, while the fraction of pores filled in the shell is equal to $S_{A}$, the fraction corresponding to the primary adsorption isotherm (that is, the amount adsorbed is $a_{A}$ ). The mean value of the fraction filled in the whole microparticle is obviously $S_{D}$, the fraction corresponding to the real primary desorption isotherm (the amount adsorbed is $a_{D}$ ). F rom the material balance on the microparticle, it is then easy to evaluate the thickness of the core $R_{1}$

$$
R_{1}=R \sqrt[3]{\frac{a_{D}-a_{A}}{a^{U}-a_{A}}}=R \sqrt[3]{\frac{S_{D}-S_{A}}{1-S_{A}}}
$$

The material balance (E q. 11) and the evaluation of the core and shell thicknesses are important also for the desorption kinetics prediction for real finite systems.

\section{Adsorption-desorption kinetics for finite systems}

The theoretical relations of M odel A 1 were developed for the idealized case of an infinite network (that is, Bethe tree), in which all pores are conductive. There are neither blind pores nor nonconductive pores, and the effect of decreasing the total mass transport rate by the influence of the flow of capillary condensate in pores completely filled by the condensate is important only for $\mathrm{x} \geq \mathrm{x}^{\mathrm{U}}$. In real porous adsorbents, there exists both blind (dead-end) pores and nonconductive pores.

The problem of nonconductive pores was discussed by R adjy (1974) and Lee and $H$ wang (1986). They assumed that in a cylindrical pore, an annular film is formed on the solid wall and considered that the thickness of this film is the same as the thickness of the adsorbed layer on a free surface not subject to capillary condensation. Therefore, it increases when the vapor concentration increases and the smallest pores may become completely nonconductive at higher relative pressures. The fraction of pores that are nonconductive will increase with increasing pressure as well as with increasing $q$.

The blind (dead-end) pores are accessible for filling by surface adsorption and capillary condensation; but, after filling with capillary condensation, they do not increase the total mass transport rate, but on the contrary, decrease it, because they do not conduct the flow. A gain, the fraction of pores that belongs to the blind clusters will increase with increasing q. For $q=1$, all pores are filled with condensate and belong to blind clusters. Therefore, in real porous adsorbents with a broad pore-size distribution and with the presence of the blind pores, all three main mechanisms (surface diffusion, capillary condensation, and liquid flow of capillary condensate) may already be operative simultaneously below point $U$. The existence of nonconductive or blind pores can explain the maximum in the concentration dependence of diffusivity during adsorption at a relative pressure $\mathrm{x}<\mathrm{x}^{\mathrm{U}}$. In our previous work (R ajniak and Y ang, 1996), a model was proposed by taking into account the empirical fraction of blind pores. U sing that previous model with an adjustable quantity $a^{*}$, it was possible to fit the experimental diffusivity data. A similar approach can be used to take into account also the existence of the nonconductive pores.

In this work we formulate a completely predictive approach based on the properties of B ethe trees. For such lattices, the analytical solution for the evaluation of the fraction of liquid filled pores without connection to infinity (exterior surface) B is available (Stinchcombe, 1974; L arson and D avis, 1982), that is,

$$
\sum_{j=2}^{C-1} B^{C-j}+\frac{q-1}{q}=0
$$

and

$$
\mathrm{Q}=1-\mathrm{B}
$$

represents the probability that an arbitrarily chosen conducting branch leaving a given site is an infinite cluster.

We will define a "blind" liquid-filled pore as one having also all its $C$ neighbors liquid filled and with all its $C$ neighbors connected to infinity (external surface) via at least one connected pathway of liquid-filled pores. The situation is shown in Figure 6. So the probability s that any pore is "blind" is given by probability $q$ (that a given pore is liquid filled) multiplied by $\mathrm{q}^{\mathrm{C}}$ (the probability that all its $C$ neighbors are also liquid filled) and multiplied by probability $Q^{\mathrm{C}}$ (that all $\mathrm{C}$ neighbors have at least one liquid-filled branch connected to infinity), that is,

$$
\mathrm{S}=\mathrm{q} \mathrm{q}^{\mathrm{C}} \mathrm{Q}^{\mathrm{C}}
$$

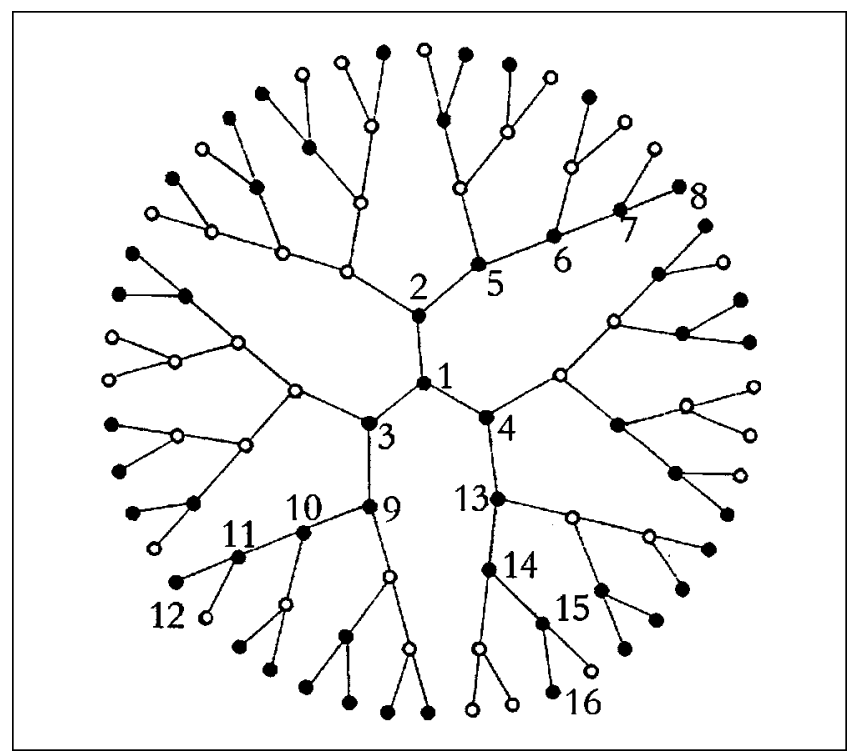

Figure 6. Dead-end (blind) pore for Bethe tree network. The filled central pore is blind because all three neighbors (pores $2,3,4)$ are filled and connected to infinity (surface) via at least one connected pathway of liquid-filled pores (that is, pathway 2-5-6-7-8, pathway 3-9-10-11-12, and pathway 413-14-15-16). 

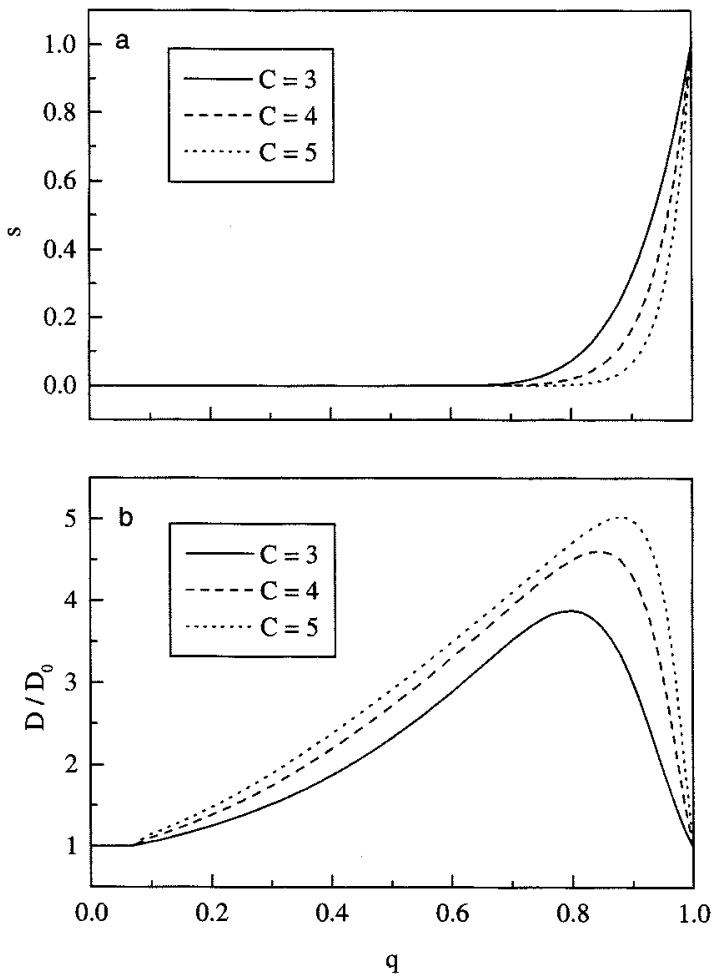

Figure 7. Adsorption kinetics for finite systems vs. fraction of pores filled.

(a) Fraction of blind pores (s) for various connectivity (C) (E qs. 12-14); (b) dependence of diffusivity for various connectivity $d_{S}=d_{F}=1, d_{C}=6$.

The theoretical dependence of the fraction of blind pores $\mathrm{s}$ as a function of $q$ is shown in Figure 7 for various values of connectivity.

The model for the adsorption kinetics for the finite (real) media incorporating the existence of the blind pores is therefore given below:

\section{Model A2:}

For $x \leq x^{L}$ :

$$
f_{1}=1 \quad f_{2}=f_{3}=0 \quad d_{E M A}=d_{S}
$$

For $\mathrm{x}^{\mathrm{L}}<\mathrm{x}<\mathrm{x}^{\mathrm{U}}$ :

$$
\begin{array}{ccc}
f_{1}=1-q & f_{2}=q(1-s) \quad f_{3}=q s \\
d_{1}=d_{s} & d_{2}=d_{c} \quad d_{3}=d_{F}
\end{array}
$$

$s$ given by solution of $E$ qs. $12-14$

$$
d_{E M A} \text { given by solution of Eq. } 9 \text { for } n=3
$$

For $\mathrm{x} \geq \mathrm{x}^{\mathrm{U}}$ :

$$
f_{1}=f_{2}=0 \quad f_{3}=1 \quad d_{E M A}=d_{F}
$$

The theoretical dependence of the effective diffusivity based on M odel A 2 for various values of connectivity is shown in Figure $7 \mathrm{~b}$ as a function of pores filled by capillary condensation q. Comparing Figures 7a and 7b, it can be concluded that the acceleration of the mass transport rate is more pronounced for higher values of connectivity where the existence of blind pores is less important. A higher value of connectivity also shifts the position of the maximum diffusivity to a higher value of concentration.

The use of EMA employed in the development of the network model for mass transfer during adsorption is based on the assumption that the various classes of pore diffusivities (conductances) are spatially distributed at random. This assumption is valid for the case of adsorption, because all the pores accessible to a given phase are effectively occupied by it. Its validity is more doubtful for desorption when the spatial topology of the phases is influenced by pore blocking. As a consequence of pore blocking for the infinite systems, there is no desorption and no mass transport for concentrations higher than that corresponding to the percolation threshold. This problem was already discussed above, and, for real finite systems, it seems to be logical that the desorption begins for the higher concentrations when evaporation proceeds from the external surface of the finite microparticle. In the second step the boundary of the phase separation along the widest pores connected with the external surface penetrates into the pellet (microparticle) volume.

For desorption kinetics for the real finite system, the following assumptions are made:

(1) There exist two basic configurations inside the microparticles of the adsorbent, that is, a completely filled core and a partially filled shell. The thickness of the core $R_{1}$ is given by Eq. 11 (see also Figure 4).

(2) For mass transport in the core, the model for infinite medium ( $M$ odel $D 1$ ) can be used, that is, there is no desorption from the core for $x>x^{\top}$.

(3) There is no pore blocking (and no hysteresis) in the shell, and mass transport in the shell occurs at all relative pressures.

Following the discussion above, the model for predicting the effective diffusivity for desorption in the finite media is summarized as follows:

\section{Model D2:}

For the whole particle:

For $\mathrm{x} \geq \mathrm{x}^{\mathrm{U}}$ :

$$
f_{1}=0 \quad f_{2}=1 \quad d_{E M A}=d_{F}
$$

For $\mathrm{x} \leq \mathrm{x}^{\mathrm{L}}$ :

$$
f_{1}=1 \quad f_{2}=0 \quad d_{E M A}=d_{S}
$$

For the core:

For $\mathrm{x}^{\top}<\mathrm{x}<\mathrm{x}^{\mathrm{U}}$ :

$$
f_{1}=0 \quad f_{2}=S_{D}=1 \quad d_{E M A, \text { core }}=0
$$

For $\mathrm{x}^{\mathrm{L}}<\mathrm{x}<\mathrm{x}^{\top}$ :

$$
\begin{gathered}
f_{1}=1-S_{D} \quad f_{2}=S_{D} \quad d_{1}=d_{S} \quad d_{2}=d_{E} \\
d_{E M A} \text {, core given by solution of Eq. } 9 \text { for } n=2
\end{gathered}
$$


For the shell:

For $\mathrm{x}^{\mathrm{L}}<\mathrm{x}<\mathrm{x}^{\mathrm{U}}$ :

$$
\begin{aligned}
& f_{1}=1-S_{A} \quad f_{2}=S_{A} \quad d_{1}=d_{S} \quad d_{2}=d_{E} \\
& d_{E M A} \text {, shell given by solution of Eq. } 9 \text { for } n=2
\end{aligned}
$$

The theoretical dependence of the effective diffusivity for both core and shell is shown in Figure 8. The dependence fore the core diffusivity is the same as that by M odel D 1 (see Figure 3b). Both diffusivities decrease with an increasing relative pressure, and they join at the two limiting points of the main hysteresis loop $L$ and $U$. H owever, there is no minimum in any of the dependencies.

From adsorption-desorption experiments (such as by a gravimetric method), we can evaluate only one apparent diffusivity which is representative of the whole microparticle. In the next part, the models of diffusion in the finite microparticle are formulated for both adsorption and desorption. Theoretical prediction of the apparent diffusivity is also discussed in the following.

\section{Models for mass transport in a single microparticle}

Processes in porous media can be studied by the continuum approach or the discrete approach. The present study refers to the discrete approach and is based on a representation of the pore space as a network of interconnected pores.
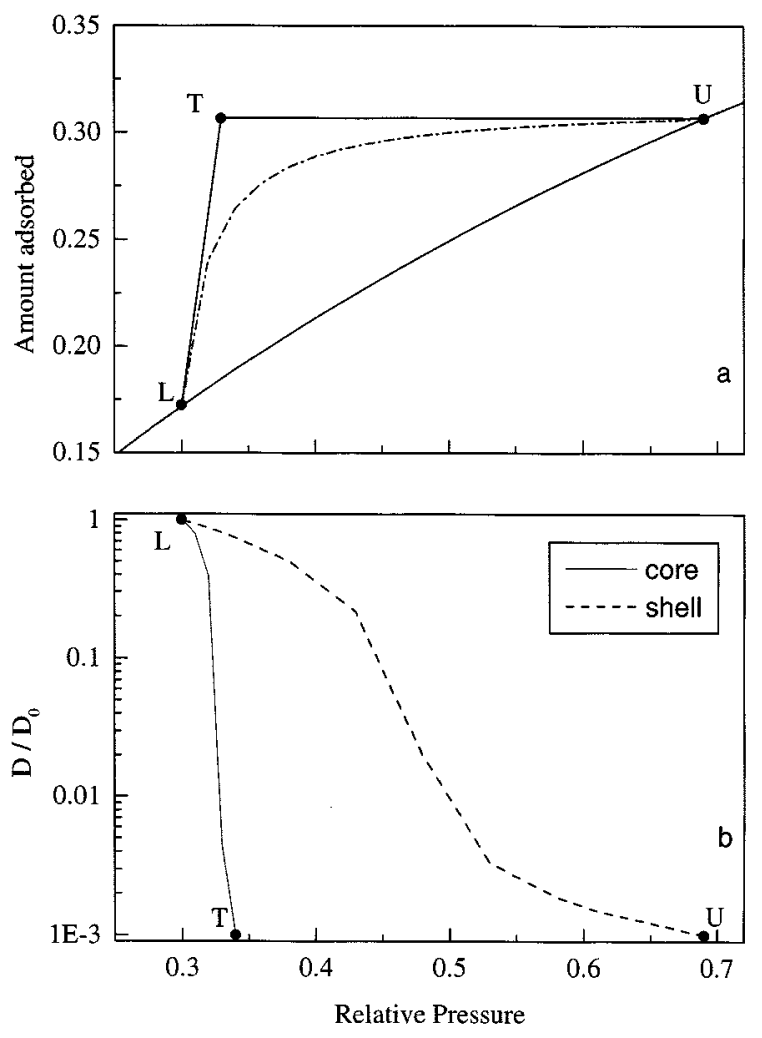

Figure 8. Theoretical prediction of diffusivities by Model D2 for system silica gel-water.

(a) E quilibria and (b) diffusivity for shell $D_{\text {shell }}$ and core $D_{\text {core }}$ computed using M odel D 2 with $d_{S}=1$ and $d_{E}=0.001$.
The effective medium approximation is then used for evaluation of the effective diffusivities of the disordered medium. Then, the continuum model based on the diffusion equation (Fick's law) can be employed. From the foregoing analysis, it is clear that effective diffusivities for adsorption and desorption are strongly concentration-dependent. However, if the uptake occurs over a small step change in the adsorbed-phase concentration, and with sufficiently high total flow rate, we may assume the absence of external heat- and mass-transfer resistances. We also assume isothermal behavior during the sorption. Then, the mass transport in a spherical microparticle with radius $R$ can be represented by the transient diffusion equation with a constant diffusivity:

\section{Model A3}

$$
\begin{aligned}
& t<0 \quad 0 \leq r \leq R \quad a=a^{0} \\
& t \geq 0 \quad r=R \quad a=a_{\infty} \\
& r=0 \quad \frac{\partial a}{\partial r}=0 \\
& 0<r<R \quad \frac{\partial a}{\partial t}=D\left(\frac{\partial^{2} a}{\partial r^{2}}+\frac{2}{r} \frac{\partial a}{\partial r}\right)
\end{aligned}
$$

The analytical or numerical solution of Eqs. $15-18$ is available (Kärger and Ruthven, 1992; R ajniak, 1985). For predictions of mass transport in the particle during adsorption, the mathematical model $A 3$ can be used with $D=D_{E M A}$ computed via M odel A 2 and Eq. 10.

This model can also be used for desorption assuming constant diffusivity in the whole microparticle. However, the prediction of mass transport during desorption is more complicated. As discussed earlier, there are various configurations of pores and, consequently, various "diffusivities" in the core and in the shell. The mathematical model then contains transient diffusion equations for the core and for the shell.

\section{Model D3}

$$
\begin{gathered}
t<0 \quad 0 \leq r<R_{1} \quad a=a_{\text {core }}^{0} \\
R_{1} \leq r \leq R \quad a=a_{\text {shell }}^{0} \\
t \geq 0 \quad r=R \quad a=a_{\alpha} \\
r=R_{1} \quad D_{E M A, \text { core }}\left(\frac{\partial a}{\partial r}\right)_{-}=D_{E M A, \text { shell }}\left(\frac{\partial a}{\partial r}\right)_{+} \\
\quad r=0 \quad \frac{\partial a}{\partial r}=0 \\
0<r<R_{1} \quad \frac{\partial a}{\partial t}=D_{E M A, \text { core }}\left(\frac{\partial^{2} a}{\partial r^{2}}+\frac{2}{r} \frac{\partial a}{\partial r}\right) \\
R_{1}<r<R \quad \frac{R^{2} a}{\partial t}=D_{E M A, \text { shell }}\left(\frac{2}{\partial r^{2}}+\frac{\partial a}{r}\right)
\end{gathered}
$$


Core and shell diffusivities are predicted by M odel D 2 and Eq. 10. The numerical solution of Eqs. $19-25$ is done using a global spline orthogonal collocation method (Villadsen, Michelsen, 1978; R ajniak, 1985). Finally, the apparent Fickian diffusivity $D_{\text {app }}$ for the whole particle is obtained by matching the solution of the Model $A 3$ with $D=D_{\text {app }}$ to the solution of M odel D3.

\section{Experimental Studies}

Vycor glass and silica gel were used in the experiments. $V$ ycor is a porous glass which has been widely used as a model material in studies of properties of fluids and molecules in highly confined geometries. The large internal surface area of $\mathrm{V}$ ycor effectively adsorbs molecules at low ambient vapor pressures, while the large pore volume effectively absorbs bulk fluids by capillary condensation at higher ambient vapor pressures (Page et al., 1993). Silica gel, Davison Grade H, a standard commercial desiccant was used in our previous experiments (R ajniak and $Y$ ang, 1993, 1994, 1996).

Sorption data for nitrogen at liquid nitrogen temperature $77 \mathrm{~K}$ were obtained using a Carlo E rba Sorptomatic 1900 apparatus for both sorbents. The Sorptomatic 1900 is a fully automatic instrument for measuring the adsorption and desorption of gases (usually nitrogen) on a solid sample. It uses a multipoint technique for determining the complete adsorption and desorption isotherms from which specific surface area (using either B.E.T. or the Dubinin equation according to the sample), and pore-size/pore-volume distributions of solids are obtained. A complete adsorption-desorption isotherm, which usually takes hours of operation, is obtained automatically.

Equilibrium results for the system silica gel-nitrogen have shown complete reversibility through the entire pressure range. O $\mathrm{n}$ the other hand, results for the system $\mathrm{V}$ ycor glassnitrogen have shown a significant hysteresis loop. The specific BET surface area and pore volume for the silica gel were determined to be $767 \mathrm{~m}^{2} / \mathrm{g}$ and $0.398 \mathrm{~cm}^{3} / \mathrm{g}$, respectively. The BET surface area and pore volume for the $V$ ycor glass were $189 \mathrm{~m}^{2} / \mathrm{g}$ and $0.715 \mathrm{~cm}^{3} / \mathrm{g}$, respectively.

For the system silica gel-water vapor, the equilibrium and the kinetic data were measured using a Mettler TA2000C Thermoanalyzer (TGA). For the kinetic data measurement, the flow conditions were adjusted to avoid or minimize influence by external diffusion. The total flow rate of the mixture flowing to the TGA was measured by using two additional gas wash bottles. The experimental conditions were adjusted to ensure that the external diffusion resistance was absent (or minimized to a negligible level) and that the wet helium stream was indeed saturated ( $R$ ajniak and $Y$ ang, 1996). The equilibrium and kinetic data for the system $V$ ycor glassnitrogen were measured using the Sorptomatic 1900 described above. There was no influence by external diffusion, because pure nitrogen was used in the experiments.

In the diffusivity measurement, the sample initially at equilibrium was subject to a sudden small change in partial pressure and the weight changes during adsorption or desorption were continually recorded. The heat effects during the sorption measurements were minimized by allowing only small step changes in relative pressure during each measurement. Because the sorption rates were independent of the pellet size, we can assume that the total sorption rate was controlled by the mass transport processes within the microparticles. Successive adsorption and desorption were conducted by changing the composition of the adsorptive and allowing adequate time to establish equilibrium.

\section{Results and Discussion}

In all figures the experimental and theoretical diffusivity data are normalized. The data are ratioed against the Fickian diffusivity at the lower closure point of the hysteresis loop $D_{0}$. The ratio $D / D_{0}$ is plotted in all figures. The mass transport process is expected to be reversible in the region of surface diffusion. Study of the concentration dependence of diffusivity below the lower limiting point $L$ and above the upper limiting point $U$ is out of the scope of this work. Evaluation of the absolute values of the diffusivities for the systems studied in this work requires information on the characteristic dimension of the microparticle in which the mass transport process occurs. The term diffusivity is also used in the formulation of the EMA model for pores of various kinds, such as for pores in which only surface diffusion occurs and for pores in which capillary condensation occurs.

The experimental rate data for adsorption are first analyzed. The data for the system silica gel-water vapor reported earlier (Rajniak and $Y$ ang, 1996) are treated with the improved network model for adsorption kinetics M odel A2. The experimental results at $298 \mathrm{~K}$ are compared in Figure 9 with the predictions from the models $A 1$ and A 2. The experimental concentration dependence of the diffusivity shows a maximum near the upper closure point $U$. In comparison to the results of $M$ odel $A 1$ for an infinite medium presented earlier (R ajniak and $Y$ ang, 1996), a significant improvement in the theoretical prediction is seen. The prediction by Model A 2 is based completely on theoretical considerations. The value of the connectivity $C=3$ was evaluated from the equilibrium data using M ason's method ( $M$ ason, 1988; R ajniak and $Y$ ang,

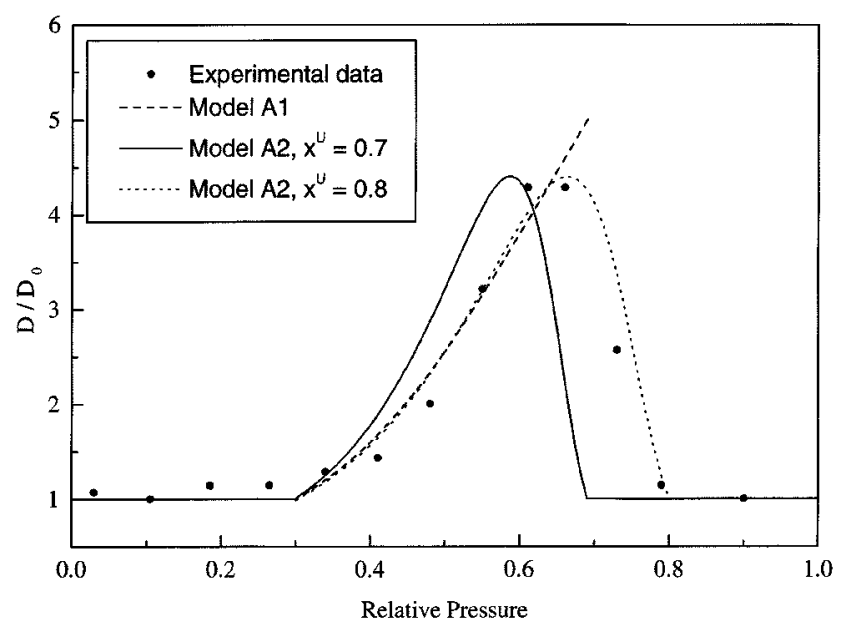

Figure 9. Experimental diffusivity vs. theoretical predictions by Models A1 and A2 for the system silica gel-water vapor at $298 \mathrm{~K}$ for the adsorption case.

$d_{\mathrm{S}}=1, \mathrm{~d}_{\mathrm{C}}=5$. 
1996). A Il the other parameters used for the theoretical prediction are the same as reported earlier, that is, positions of the limiting points of the main hysteresis loop and parameters of the primary adsorption isotherm. The only adjustable parameter for the diffusivity prediction by Model A 2 is the ratio of the diffusivity in the capillary condensation region and that of the surface diffusivity, that is, $d_{d} / d_{s}$. No adjustable parameters similar to $x^{*}$ (the empirical relative pressure at which the blind pores become filled with capillary condensate ( $R$ ajniak and $Y$ ang, 1996) are needed here.

The analytical relations (E qs. 12-14) for the evaluation of the fraction of "blind" pores are derived using the Bethe tree lattice as the model for the pore network. This is the main advantage of the Bethe lattice, as it is often possible to derive analytical formulas for the properties of interest; and often the predictions of such formulas agree surprisingly well with those of three-dimensional systems (Sahimi, 1995). As discussed also by Mason (1988), general forms of the percolation properties that are known for both crystal structures and Bethe trees show surprising similarities.

The use of EMA in the development of the network model for mass transfer during adsorption is based on the assumption that the various classes of pore diffusivities (conductances) are spatially distributed at random. This assumption is valid for the case of adsorption, because all the pores accessible to a given phase are effectively occupied by it. The approach derived here solves the main artifact of our earlier model (Rajniak and $Y$ ang, 1996), that is, a discontinuity in the concentration dependence of diffusivity.

$M$ odel $A 2$ represents the next step in the unification of the equilibrium theory based on the pore-blocking interpretation of hysteresis and the percolation model of mass transport in the network of pores randomly filled by capillary condensate or surface adsorption. U sing M odel A3, the mass transport process in the particle can be predicted using diffusivity values calculated from Model A 2 .

The main objective of the present work is to develop and check the validity of the network model for the difficult problem of desorption kinetics, which has not been done previously. Experimental kinetic data available in the literature for the capillary condensation regime ( $R$ him and $H$ wang, 1975; T amon et al., 1981; Toei et al., 1983; E berly and Vosberg, 1965) cannot be compared with predictions from our models because either the equilibrium data are incomplete, are missing, or the kinetics data are for the adsorption case only. Therefore, it was necessary to measure new sets of complete equilibrium and kinetic data. Two systems, silica gelwater vapor and Vycor glass-nitrogen, were studied. The complete equilibrium data for the system silica gel-water vapor were published elsewhere (R ajniak and $Y$ ang, 1993, 1994). The kinetic data for adsorption are analyzed above (see Figure 9).

In Figure 10 the experimental data of the relative Fickian diffusivity for desorption for the system silica gel-water vapor are shown and are compared to the theoretical predictions. The experimental concentration dependence of the diffusivity shows a minimum near the lower closure point L. U sing the equilibrium data and following the theory presented in the foregoing, M odel D 2 is used to predict diffusivities in the core and in the shell. M odel D 3 uses predictions from M odel D 2 for calculating the core and shell diffusivities and subse-
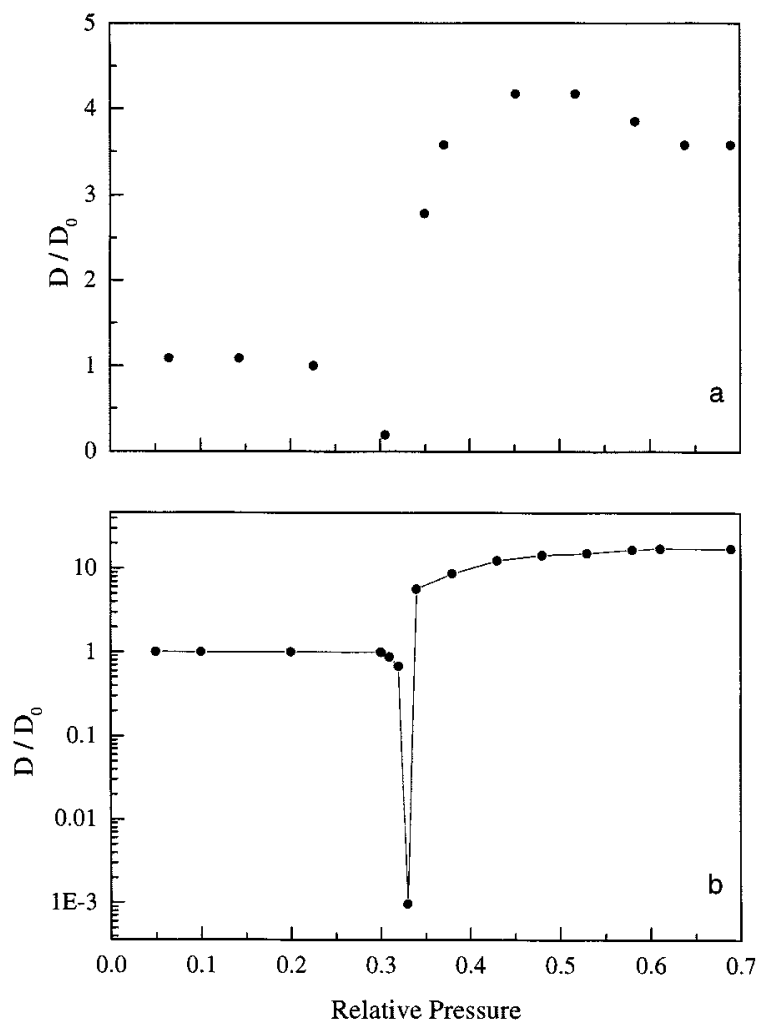

Figure 10. Experimental diffusivity data (a) vs. theoretical predictions (b) by using Model D3 for the system silica gel-water vapor at $298 \mathrm{~K}$, for the desorption case.

$\mathrm{d}_{\mathrm{S}}=1 ; \mathrm{d}_{\mathrm{E}}=0.001$.

quently yields the overall diffusivity for the whole particle. Starting from the concentration (or relative pressure) $\mathrm{x}=\mathrm{x}^{\mathrm{U}}$ and decreasing relative pressure by small changes, we solve $M$ odel $D 3$ for $D_{E M A}$, core and $D_{E M A}$, shell that are predicted by $M$ odel D2. By matching the solution of Model D 3 to the soIution of the diffusion equation (M odel A 3 is for the desorption case), we obtain the overall value $D$ for each transient. The results are normalized by dividing by the diffusivity $D_{0}$.

For relative pressures $\mathrm{x}>\mathrm{x}^{\top}$, desorption (evaporation) occurs only from this shell at the microparticle surface. The thickness of the shell increases with decreasing $x$. Both diffusivities $D_{\text {core }}$ (for the completely saturated core) and $D_{\text {shell }}$ (for the partially saturated shell) increase with decreasing pressure (see Figure $8 b$ ), but the overall diffusivity $D$ for the whole particle decreases in this region. This is because desorption proceeds in an increasingly thicker shell (with thickness $R_{1}$ given by $E q .11$ ), and the corresponding diffusion path increases. It is seen that the increasing diffusion path dominates over the accelerating effect of the shell and core diffusivities. When the concentration is dropped below $x^{\top}$, desorption starts also from the core and the diffusivity passes through a minimum, as shown in Figure 10. The mass transport (desorption) is very slow in this region and an unusual transient behavior occurs. In this stage the process is very slow, first, because desorption (evaporation) occurs from the whole core, that is, the mass-transfer path for the core molecules is the longest, and secondly, because the evapora- 
tion (which is slow process alone) occurs from the maximum amount of pores and in the pore space with the minimum number of empty pores. (A Iso, the mass-transfer path in the core alone is the longest and curved, because of only few empty pores, that is, high tortuosity.) For relative pressures smaller than threshold relative pressure, the desorption (evaporation) occurs already from the whole microparticle. There is still pore blocking, but the fraction of empty (nonblocking pores) is increasing with decreasing pressure, that is, the apparent diffusivity is increasing. This branch is nicely predicted from the theoretical relations for infinite medium (no surface effects), as shown in Figure $3 b$.

The theoretical and experimental results are in qualitative agreement. The theoretical model successfully predicts the position of the minimum in diffusivity between points $T$ and $L$. The model also predicts higher values for the overall, apparent diffusivity in the concentration range between points $U$ and $T$, compared to the diffusivities between points $T$ and $\mathrm{L}$. However, quantitative agreement is not satisfactory. The maximum experimental relative diffusivity is 4.2 and the minimum experimental diffusivity is 0.19 , while the theoretical maximum relative diffusivity is 17.4 and the minimum is 0.001 .

In Figure 11 typical dependencies of the amount adsorbed during desorption for the system silica gel-water are compared for concentrations higher and lower than the percolation threshold $x^{\top}$. The desorption (evaporation) process at the percolation threshold (close to the lower limiting point $L$ )
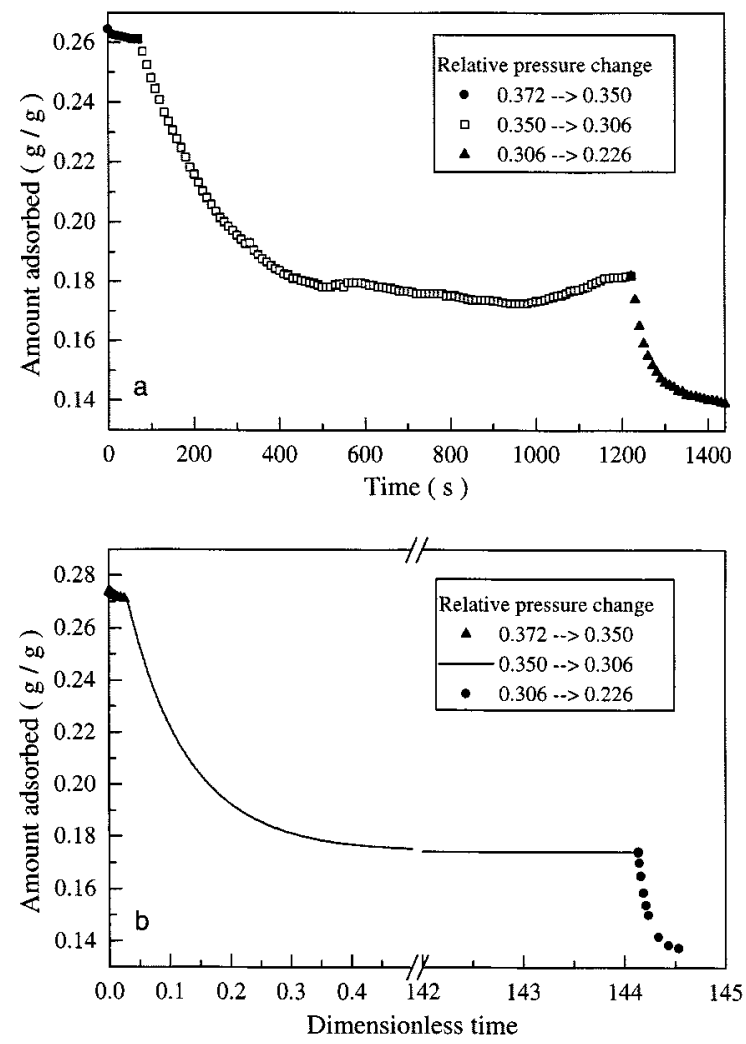

Figure 11. Desorption upon step changes in relative pressure for the system silica gel-water at 298 K.

(a) Experimental; (b) predictions by M odel D 3 .

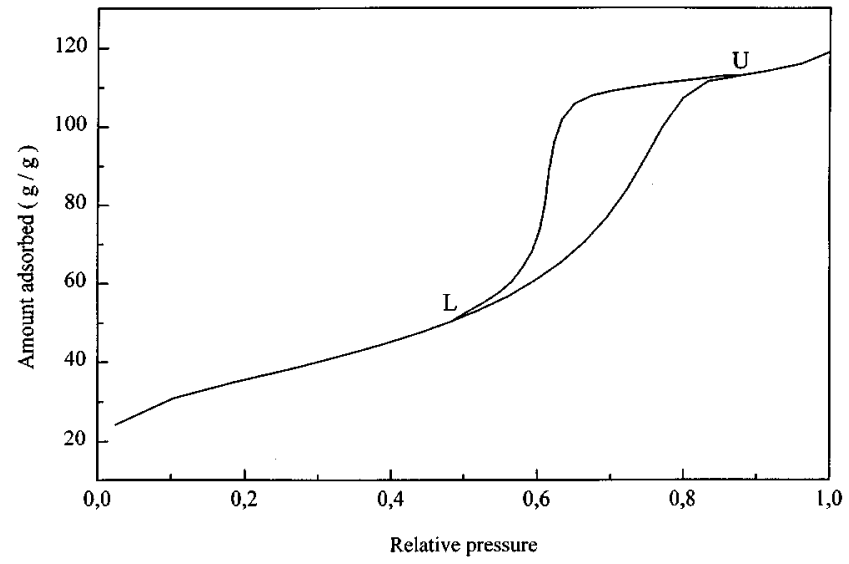

Figure 12. Experimental adsorption-desorption equilibrium data for the system Vycor glass-nitrogen at $77 \mathrm{~K}$.

is unusually slow. Also, the shape of the transient amount adsorbed at this point (for the relative pressure change from 0.35 to 0.306 ) is different from the Fickian behavior observed at other concentrations. The mass transport at this point involves a combination of various mechanisms (surface diffusion, evaporation, condensation, and liquid flow), and modeling the mass transport process by the diffusion equation is an oversimplification.

Figure 12 shows the experimental data for adsorptiondesorption equilibria for the system $V$ ycor glass-nitrogen at $77 \mathrm{~K}$. The primary adsorption isotherm of type IV and the primary desorption isotherm of type $\mathrm{H} 2$ are observed.

In Figure 13 the experimental data of the relative Fickian diffusivity for desorption for the system $V$ ycor glass-nitrogen are shown and are compared with the theoretical predictions. The behavior of the system is qualitatively similar to that of silica gel-water, that is, with a decreasing diffusivity going from point $U$ to point $T$, passing through a minimum near the threshold point $T$, followed by an increasing diffusivity between points $T$ and $L$. Compared with the results for the system silica gel-water, it was possible to measure more experiments data points between points $T$ and $L$.

The theoretical model is again able to predict well, qualitatively, the concentration dependence of the diffusivity, but the quantitative agreement is understandably poor. The maximum experimental relative diffusivity is 1.92 and the minimum experimental diffusivity is 0.13 , while the theoretical maximum relative diffusivity is 18.34 and the minimum is 0.046 .

There are several possible reasons for the quantitative discrepancy between the theory and experiment for the case of desorption kinetics:

(1) The boundary between the shell and the core is assumed to be distinct in the theoretical model, as shown in Figure 5 . In the real microparticle the boundary is a diffuse one. A $n$ improved model with a third (middle) zone between the core and the shell should better describe the reality. The mass transport path will be longer for molecules from the middle zone. As a consequence, the apparent diffusivity will be smaller for the descending branch of the diffusivity dependence. A nother consequence will be an acceleration of 

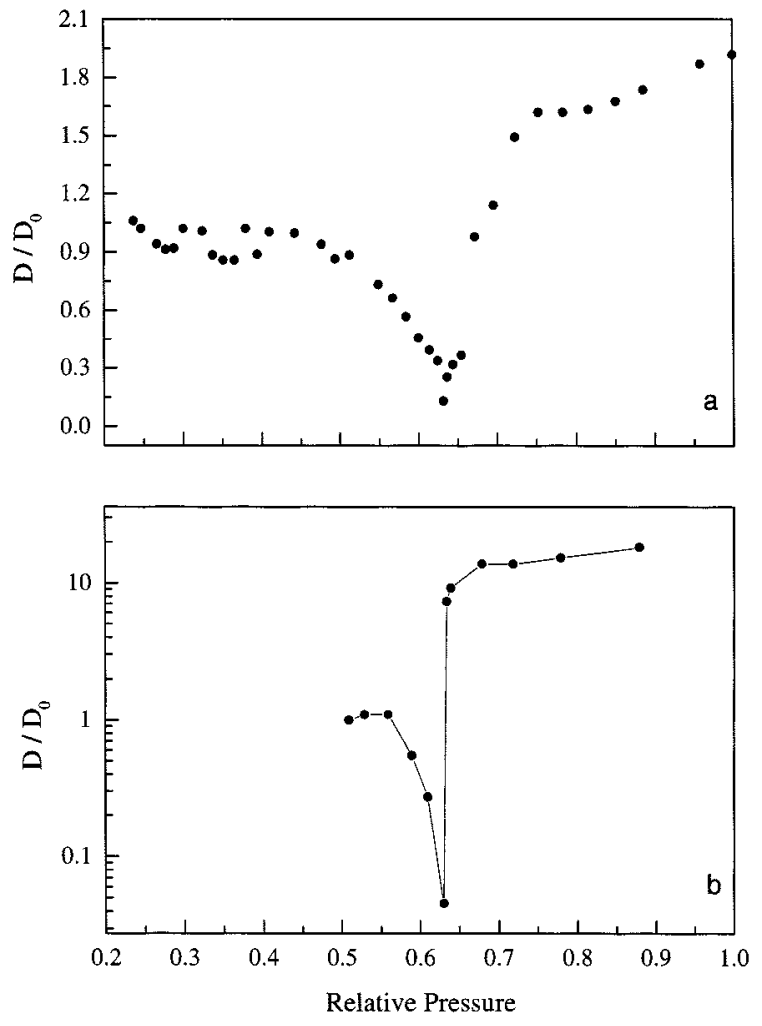

Figure 13. Experimental desorption diffusivity data.

(a) $V$ s. theoretical predictions; (b) by using M odel D 3 for the system $V$ ycor glass-nitrogen at $77 \mathrm{~K} . \mathrm{d}_{\mathrm{S}}=1, \mathrm{~d}_{\mathrm{E}}=0.001$.

the process for relative pressure $\mathrm{x} \leq \mathrm{X}^{\top}$, because slow evaporation will occur from the smaller core. So, the addition of the middle zone should decrease the maximum and increase the minimum of the theoretical dependence of diffusivity without changing the correct position of the minimum.

(2) The use of EMA in the development of the network model for mass transfer in the shell assumed that various classes of pore diffusivities (conductances) are randomly distributed in the shell. This assumption is valid for the case of adsorption, because all the pores accessible to a given phase are effectively occupied by it. It is less so for desorption. The spatial topology of the phases is influenced by pore blocking, and the distribution of phases is not random, especially in the shell for concentrations higher than the percolation threshold.

(3) The model assumes only surface diffusion and evaporation during desorption in the hysteresis region and is thus much simplified. From the theoretical and experimental study of A beles et al. (1991), different flow regimes can exist depending on the magnitude of the vapor and/or liquid pressures at the inlet and outlet sides of the porous medium. R esults of Laurindo and Prat (1996) indicate that film flows could be important in evaporation and, more generally, in phase change phenomena in porous media. Other possible complicating effects are nucleation (Parlar and Y ortsos, 1989), decompression of the condensed liquid (M ason, 1988), and flow of conductance by gravity (Prat, 1995).

(4) Simultaneous evaporation and condensation processes during the emptying of the pores of various dimensions com- bined with the actual nonisothermality can slow down the process.

(5) There exist possible loops in the path of mass transport during desorption, because of nonrandom distribution of phases. It is known that if a particular percolation property depends on the existence of loops, then the use of the Bethe tree would not be appropriate.

(6) The assumption that the diffusivities (or conductances) of the pores of various dimensions are constant could be an oversimplification.

The study of equilibria and kinetics for adsorption-desorption systems with hysteresis shows that the concepts of percolation theory play a prominant role. Theoretical predictions are excellent for simpler percolation processes, that is, desorption equilibria (bond percolation) and adsorption kinetics (ordinary percolation). Qualitative agreement is also obtained for desorption kinetics (invasion percolation), but quantitative agreement is not satisfactory for this complicated process of the invasion percolation type. For such processes, further studies are needed. Numerical M onte Carlo simulation of the percolation processes on a more realistic (but more complex) pore network, such as the diluted simple-cubic lattice, should improve the agreement between model and experiment. However, for engineering purposes (such as within a process simulation), it is always useful to have a quick solution that is capable of predicting limiting behaviors of the process, as given in this contribution.

\section{Conclusion}

$\mathrm{N}$ etwork models are formulated for predicting the concentration dependence of the Fickian diffusivity for systems exhibiting hysteresis in adsorption-desorption equilibria.

Adsorption-desorption equilibria and kinetics are studied both experimentally and theoretically. The main results are as follows:

(1) For adsorption, the network model of mass transport of condensable vapors in porous media based on the combination of the percolation model of mass transport in the pore network with the effective medium approximation ( $R$ ajniak and $Y$ ang, 1996) is further improved. A new predictive relation based on the properties of $B$ ethe lattices is proposed to account for the existence of liquid-filled "blind" pores that decrease the total mass transport rate.

(2) For desorption, a new "shell and core" (or shrinking core) picture of the network model is proposed. Information from adsorption-desorption equilibria are used to compute the thickness of the shell in which desorption/evaporation occurs for concentrations higher than the percolation threshold $\mathrm{x}^{\top}$. For concentrations lower than $\mathrm{x}^{\top}$, the model similar to the model for adsorption is used.

(3) Complete sets of experimental kinetic data for the systems silica gel-water vapor and $V$ ycor glass-nitrogen are measured and analyzed.

(4) The adsorption model successfully predicts the experimental data with a maximum in diffusivity. The desorption model correctly predicts the dependence of diffusivity with a minimum, but the quantitative agreement is not satisfactory due to a number of reasons.

(5) Improvement of the models is possible by including more transport mechanisms (such as flow of liquid conden- 
sate, nucleation) and/or by allowing the diffusivities to be dependent on concentration and pore size.

(6) The two models proposed in this work represent a unification of the theories for equilibrium and kinetics for systems with hysteresis. Information on the adsorptiondesorption equilibria is needed for the models for mass transport. A minimum number of adjustable parameters are used in the models.

\section{Acknowledgments}

We would like to thank A. H. D anzberger, P.E., for helpful discussion. The work was supported by $\mathrm{N}$ ational Science Foundation grant CTS-9520328.

\section{Notation}

$\mathrm{a}=$ amount adsorbed $(\mathrm{g} / \mathrm{g})$

$\mathrm{B}=$ fraction of liquid filled pores, Eq. 12

$\mathrm{D}=$ diffusivity in the network of pores; effective Fickian or overall diffusivity

$D_{0}=$ surface diffusivity or diffusivity at zero loading

$f(d)=$ distribution function, Eq. 8

$f(x)=$ equilibrium function, $E q .3$

$\mathrm{q}=$ site filling probability, Eq. 1

$\mathrm{Q}=$ probability, Eq. 13

$\mathrm{R}_{1}=$ shell thickness

$\mathrm{t}=$ time

\section{Subscripts}

$$
\begin{aligned}
\mathrm{E} & =\text { evaporation } \\
\mathrm{F} & =\text { flow of capillary condensate } \\
\mathrm{S} & =\text { surface diffusion } \\
\infty & =\text { value at surface } \\
1,2, \ldots, \mathrm{n} & =\text { different pores }
\end{aligned}
$$

\section{Superscripts}

$T=$ percolation threshold

$0=$ initial value

\section{Literature Cited}

A beles, B., L. F. Chen, J. W. Johnson, and J. M. Drake, "Capillary Condensation and Surface Flow in Microporous Vycor Glass," Israel J. Chem., 31, 99 (1991).

Benzoni, J., and H. C. Chang, "Effective Diffusion in Bi-disperse Media-An Effective Medium A pproach," Chem. Eng. Sci., 39, 167 (1984).

Broadbent, S. R ., and J. M. H ammersley, "Percolation Processes: I. Crystals and M azes," Proc. Camb. Phil. Soc., 53, 629 (1957).

Brunovská, A., J. Ilavsky, and H. Kukurucová, "The Shell Progressive M odel of A dsorption in a Single Particle," Collect. Czechoslov. Chem. Commun., 50, 1341 (1985).

Burganos, V. N., and S. V. Sotirchos, "Diffusion in Pore Networks: Effective Medium Theory and Smooth Field Approximation," AIChE J., 33, 1678 (1987).

Büssing, W., H.-J. Bart, and R. Germerdonk, "I sothermal Liquid Transport in Porous M edia: Capillary H ysteresis Effects," Int. J. H eat M ass Transfer, 39, 1925 (1996).

Burgess, C. G. V., D. H. Everett, and S. Nuttall, "A dsorption H ysteresis in Porous M aterials," Pure A ppl. Chem., 61, 1845 (1989).

Carman, P. C., "Diffusion and Flow of Gases and Vapors Through Micropores: IV. Flow of Capillary Condensate," Proc. R. Soc. L ondon, A211, 526 (1952).

Chen, Y. D., and R. T. Y ang, "Surface Diffusion of M ultilayer Adsorbed Species," AIChE J., 39, 599 (1993).

Daian, J.-F., "From Pore-Size Distribution to Moisture Transport Properties: Particular Problems for Large Pore-Size D istributions," Drying '92, A. S. M ujumdar, ed., Elsevier, A msterdam, 263 (1992).

E berly, P. E., and D. B. V osberg, "Diffusion of Benzene and Inert
Gases Through Porous M edia at Elevated Temperatures and Pressures," Trans. Faraday Soc., 61, 2724 (1965).

E verett, D. H., Adsorption H ysteresis, The Solid-G as Interface, V ol. 2, E. A. Flood, ed., M arcel Dekker, New Y ork, p. 1055 (1967).

Flood, E. A., R. H. Tomlinson, and A. E. Leger, "The Flow of Fluids through A ctivated Carbon R ods III. The Flow of A dsorbed Fluids," Can J. Chem., 30, 389 (1952).

Gilliland, E. R., R. F. Baddour, and J. L. R ussell, "R ates of Flow Through M icroporous Solids," AIChE J., 4, 90 (1958).

Gregg, S. J., and K. S. Sing, Adsorption Surface Area and Porosity, 2nd ed., A cademic Press, London (1982).

Guyer, R. A., and K. R. M CCall, "Capillary Condensation, Invasion Percolation, Hysteresis, and Discrete Memory," Phys. Rev. B, 54, 18 (1996).

H aynes, J. M ., and R. J. M iller, "Surface Diffusion and V iscous Flow During Capillary Condensation," Adsorption at the Gas-Solid and Liquid-Solid Interface, J. R ouquerol and K. S. W. Sing, eds., Elsevier, A msterdam, p. 439 (1982).

Heinrichs, J., and N. Kumar, "Simple Exact Treatment of Conductance in a R andom Bethe Lattice," J. Phys. C.: Solid State Phys., 8, L510 (1975).

Kainourgiakis, M. E., E. S. Kikkinides, A. K. Stubos, and N. K. Kanellopoulos, "Adsorption-D esorption Gas Relative Permeability through M esoporous M edia-Network Modelling and Percolation Theory," Chem. Eng. Sci., 53, 2353 (1998).

Kapoor, A., R. T. Y ang, and C. Wong, "Surface Diffusion," Catal. Rev. Sci. Eng., 31, 129 (1989).

Kärger, J., and D. M. R uthven, Diffusion in Zeolites and Other Microporous Solids, Wiley, N ew Y ork (1992).

Kirkpatrick, S., "Classical Transport in Disordered Media: Scaling and Effective Medium Theories," Phys. Rev. Lett., 27, 1722 (1971).

Larson, R. G., and H. T. Davis, "Conducting Backbone in Percolating Bethe Lattices," J. Phys. C.: Solid State Phys., 15, 2327 (1982).

Laurindo, J. B., and M. Prat, "Numerical and Experimental N etwork Study of Evaporation in Capillary Porous Media. Phase Distributions," Chem. Eng. Sci., 51, 5171 (1996).

Lee, K. H., and S. T. H wang, "The Transport of Condensable Vapors Through a Microporous V apor G lass M embrane," J. Colloid Interf. Sci., 110, 544 (1986).

Lee, S. Y., and R. A ris, "The Distribution of Active Ingredients in Supported Catalysts Prepared by Impregnation," Cata. Rev.-Sci. Eng., 27, 207 (1985).

Li, J.-C., D. K. R oss, and M. J. Benham, "Small-A ngle Neutron Scattering Studies of Water and Ice in Porous V ycor Glass," J. Appl. Cryst., 24, 794 (1991).

Li, X., and Y. C. Y ortsos, "V isualization and Simulation of Bubble Growth in Pore Networks," AIChE J., 41, 214 (1995).

Lilly, M. P., P. T. Finley, and R. B. H allock, "M emory, Congruence, and $\mathrm{A}$ valanche E vents in $\mathrm{H}$ ysteretic Capillary Condensation," Phys. Rev. L ett., 71, 4186 (1993).

Liu, H., L. Z hang, and N. A. Seaton, "A nalysis of Sorption H ysteresis in M icroporous Solids U sing a Pore N etwork M odel," J. Colloid Interf. Sci., 156, 285 (1993).

M aneval, J. E., M. J. M CC arthy, and S. Whitaker, "Studies of the D rying Process by N M R Imaging," D rying, E Isevier, A msterdam, $p$. 170 (1991).

Markos, J., A. Brunovská, and J. Ilavsky, "Modelling of Catalytic R eactors with Catalyst D eactivation: IV. Parameter Estimation of the Rate Equations of Heterogeneous Catalyst Deactivation," Chem. Papers, 41, 375 (1987).

$M$ ason, G., "D etermination of the Pore/Size Distribution and PoreSpace Interconnectivity of $\mathrm{V}$ ycor Porous G lass from AdsorptionD esorption H ysteresis Capillary Condensation I sotherms," Proc. R. Soc. L ondon, A415, 453 (1988).

$\mathrm{N}$ aono, H., and M. H akuman, "A nalysis of Porous Texture by $M$ eans of Water V apor A dsorption I sotherm with Particular A ttention to L ower Limit of H ysteresis L oop," J. Coll. Interf. Sci., 158, 19 (1993).

Neimark, A. V., L. I. K heifez, and V. B. Fenelonov, "Theory of Preparation of Supported Catalysts," Ind. Eng. Chem. Prod. Res. Dev., 20, 439 (1981).

Page, J. H., J. Liu, B. A beles, H. W. Deckman, and D. A. Weitz, "Pore-Space Correlations in Capillary Condensation in Vycor," Phys. Rev. L ett., 71, 1216 (1993).

Parlar, M., and Y. C. Y ortsos, "Percolation Theory of Vapor Ad- 
sorption-D esorption Processes in Porous M aterials," J. Colloid Interf. Sci., 124, 162 (1988).

Parlar, M., and Y. C. Y ortsos, "Nucleation and Pore Geometry Effects in Capillary Desorption Processes in Porous M edia," J. Colloid Interf. Sci., 132, 425 (1989).

Pel, L., H. Brocken, and K. Kopinga, "D etermination of M oisture Diffusivity in Porous Media U sing M oisture Concentration Profiles," Int. J. Heat Mass Transfer, 39, 1273 (1996).

Prat, M., "Percolation M odel of D rying under I sothermal Conditions in Porous M edia," Int. J. Multiphase Flow, 19, 691 (1993).

Prat, M., "I sothermal D rying of $\mathrm{Non}-\mathrm{H}$ ydroscopic Capillary-Porous Materials as an Invasion Percolation Process," Int. J. Multiphase Flow, 21, 875 (1995).

R adjy, F., "M oisture Transport in M icroporous Substances," J. Mat. Sci., 9, 744 (1974).

$\mathrm{R}$ ajniak, P., "A nalysis of a O ne-Component Sorption in a Single A dsorbent Particle by the Orthogonal Collocation M ethod. IV. O nePoint Collocation $M$ ethod and Linear D riving-Force A pproximation," Chem. Papers, 39, 447 (1985).

R ajniak, P., and R. T. Y ang, "A Simple Model and Experiments for Adsorption-Desorption Hysteresis: Water Vapor on Silica Gel," AIChE J., 39, 774 (1993).

Rajniak, P., and R. T. Y ang, "H ysteresis-D ependent AdsorptionDesorption Cycles: Generalization for Isothermal Conditions," AIChE J., 40, 913 (1994).

R ajniak, P., and R. T. Y ang, "U nified N etwork M odel for Diffusion of Condensable V apors in Porous M edia," AIChE J., 42, 319 (1996).

R him, H ., and S. T. H wang, "Transport of Capillary Condensate," J. Coll. Interface Sci., 52, 174 (1975).

Sahimi, M., "Nonlinear Transport Processes in Disordered M edia," AIChE J., 39, 369 (1993).

Sahimi, M., Applications of Percolation Theory, Taylor and Francis, London (1994).

Sahimi, M ., Flow and Transport in Porous M edia and Fractured Rock, $\mathrm{VCH}$ V erlagsgesellschaft mbH, D-69451 Weinheim (1995).

Sahimi, M., G. R. G avalas, and T. T. Tsotsis, "Statistical and Contin- uum M odels of Fluid-Solid R eaction in Porous M edia,"'Chem. Eng. Sci., 45, 1443 (1990).

Seaton, N. A., "Determination of the Connectivity of Porous Solids from Nitrogen Sorption M easurements," Chem. Eng. Sci., 46, 1895 (1991).

Stauffer, D., and A. A harony, Introduction to Percolation Theory, 2nd ed., Taylor and Francis, London (1992).

Stinchcombe, R. B., "Conductivity and Spin-Wave Stiffness in Disordered Systems-A n Exactly Soluble M odel," J. Phys. C.: Solid State Phys., 7, 179 (1974).

Tamon, H., M. O kazaki, and R. Toei, "Flow Mechanism of Adsorbate Through Porous M edia in Presence of Capillary Condensation," AIChE J., 27, 271 (1981).

Toei, R ., H. Imakoma, H. Tamon, and M. O kazaki, "W ater Transfer Coefficient in A dsorptive Porous Body,"J. Chem. Eng. Japan, 16, 364 (1983).

U hlhorn, R . J . R ., K. K eizer, and A . J. Burgraaf, "G as Transport and Separation with Ceramic Membranes I. Multilayer Diffusion and Capillary Condensation," J. Memb. Sci., 66, 259 (1992).

Villadsen, J., and M. L. M ichelsen, Solution of Differential Equation Models by Polynomial Approximation, Prentice $\mathrm{Hall}$, Englewood Cliffs, NJ (1978).

Weisz, P. B., "Diffusion Transport in Chemical Systems-Key Phenomena and Criteria, Berichte der Bunsen-Gesselschaft," Phys. Chem., 79, 798 (1975).

Wilkinson, D., and J. F. Willemsen, "Invasion Percolation: A New Form of Percolation Theory," J. Phys. A, 16, 3365 (1983).

$Y$ ang, R. T., G as Separation by Adsorption Processes, Butterworth, Boston (1987) [in paperback, Imperial College Press and World Scientific Publishers, River E dge, NJ (1997)].

Y ortsos, Y . C., C. Satik, J.-C. Bacri, and D. Salin, "L arge-Scale Percolation Theory of Drainage," Transport in Porous M edia, 10, 171 (1993).

Manuscript received Sept. 2, 1998, and revision received Jan. 19, 1999. 